\title{
Microbiota intestinal y estreñimiento. Una puesta al día
}

\section{Intestinal microbiota and constipation. An update}

\author{
Ignacio Hanna-Jairala ${ }^{1}$ y Luis M. Bustos-Fernández ${ }^{2}$
}

${ }^{1}$ Servicio de Gastroenterología, Hospital Alcívar, Guayaquil, Ecuador; ${ }^{2}$ Centro Médico Bustos-Fernández, Buenos Aires, Argentina

\section{RESUMEN}

La microbiota intestinal y su influencia sobre los trastornos funcionales gastrointestinales han sido ampliamente estudiadas en los últimos años, pero parece que la evidencia en relación a microbiota y trastornos funcionales que cursan con estreñimiento es menor que la referente a la diarrea como síntoma predominante. En esta revisión presentamos la evidencia más relevante en relación con la influencia de los cambios en la composición de la microbiota intestinal y la presencia de estreñimiento, como la abundancia de Methanobrevibacter smithii, así como también los resultados de las distintas intervenciones para modular la microbiota intestinal como alternativas terapéuticas para los pacientes con trastornos funcionales con predominio de estreñimiento, tales como cambios de la alimentación que contemplan la suplementación con fibra o dietas específicas como la baja en FODMAP (oligosacáridos, disacáridos, monosacáridos y polioles fermentables), el uso de prebióticos como fructooligosacáridos, inulina, lactulosa o lactilol, la utilización de probióticos, principalmente las fórmulas que contienen Bifidobacterium lactis DN-173010 y Bifidobacterium lactis HN019, Lactobacillus reuteri y Lactobacillus casei Shirota, el tratamiento con antibióticos como rifaximina o neomicina, y el trasplante de microbiota fecal.

Palabras clave: Antibióticos. Dieta. Estreñimiento. Microbiota. Probióticos. Trasplante de microbiota fecal.

\section{ABSTRACT}

The intestinal microbiota and its influence on gastrointestinal functional disorders has been widely studied in recent years, however it seems that the evidence regarding microbiota and functional disorders with constipation is less than that referring to those that present with diarrhea such as predominant symptom. In this review we present the most relevant evidence regarding the influence of changes in the composition of the intestinal microbiota and the presence of constipation such as the abundance of Methanobrevibacter smithii, as well as the results of the different interventions to modulate the intestinal microbiota as therapeutic alternatives for patients with functional disorders with a predominance of constipation such as changes in diet that include fiber supplementation or specific diets such as low FODMAPs, use of prebiotics such as fructoligosaccharides, inulin, lactulose or lactilol, use of probiotics mainly species of Bifidobacteria and Lactobacilli, treatment with antibiotics such as rifaximin or neomycin and fecal microbiota transplantation.

Key words: Antibiotics. Diet. Constipation. Microbiota. Probiotics. Fecal microbiota transplantation.

\author{
Correspondence to: \\ *Ignacio Hanna-Jairala \\ E-mail: ihann@hotmail.com
}

NeuroGastroLatam Rev. 2020;4:144-158

Received in original form: 04-04-2020

Accepted in final form: 21-09-2020

DOI: 10.24875/NGL.20000011

2462-7011/@ 2020 Sociedad Latinoamericana de Neurogastroenterología, A.C. Published by Permanyer. This is an open access article under the CC BY-NC-ND license (http://creativecommons.org/licenses/by-nc-nd/4.0/). 


\section{INTRODUCCIÓN}

La microbiota intestinal ha sido estudiada ampliamente en los últimos años y se pueden encontrar artículos relacionados con los mecanismos por los que su composición y diversidad influyen en la aparición de trastornos digestivos y extradigestivos, y también de cómo su manipulación puede ser clave para solucionarlos ${ }^{1,2}$. Hemos realizado una búsqueda en la base de datos PubMed utilizando los términos "gut microbiota" y hemos clasificado los artículos según el año de publicación entre 2005 y 2019, observando un crecimiento exponencial en la producción bibliográfica (Fig. 1). Sin embargo, este aumento en el número de publicaciones no parece haber sido equitativo en cuanto al campo de estudio. En función de esto, nuestro primer objetivo ha sido comparar el número de estudios publicados sobre la composición y la manipulación de la microbiota en la diarrea y en el estreñimiento. Es así que al hacer una búsqueda en PubMed de artículos publicados hasta el año 2019 incluyendo los términos "microbiota \& diarrhea" aparecen 1528 referencias, contra apenas 378 que aparecen con los términos "microbiota \& constipation"; estos datos evidencian que las publicaciones sobre microbiota intestinal en relación con la diarrea superan cuatro veces a las relacionadas con el estreñimiento (Fig. 2).

Basándonos en esta diferencia en cuanto al número de publicaciones, como segundo objetivo hemos querido revisar la mejor evidencia disponible sobre la microbiota y el estreñimiento, comenzando por los mecanismos que influyen en la composición de la microbiota como causa del enlentecimiento del tránsito intestinal y los síntomas de los trastornos funcionales con predominio de estreñimiento (síndrome de intestino irritable [SII]) con estreñimiento y estreñimiento funcional crónico) ${ }^{3}$, para luego repasar las estrategias utilizadas en la modulación de la microbiota intestinal como tratamiento de estos trastornos (dieta, prebióticos, probióticos, antibióticos y trasplante de microbiota fecal) y las consecuencias que conllevan estas intervenciones ${ }^{3,4}$.

Nuestro trabajo es una revisión narrativa elaborada a partir de datos relevantes encontrados en artículos seleccionados en una búsqueda en PubMed priorizando los resultados de revisiones sistemáticas, metaanálisis y ensayos controlados aleatorizados, así como también revisiones narrativas publicadas en revistas especializadas.

\section{MICROBIOTA Y TRASTORNOS FUNCIONALES CON PREDOMINIO DE ESTREÑIMIENTO}

La microbiota intestinal se compone en un $90 \%$ por bacterias cuyos filotipos predominantes son Firmicutes y Bacteroidetes; el 10\% restante se reparte mayoritariamente entre Actinobacterias y Proteobacterias, y en menor proporción entre Fusobacterias, Verrucomicrobia, Cianobacterias y microorganismos no bacterianos, como arqueas, virus y levaduras ${ }^{1}$.

De acuerdo con el Consenso de Roma IV, los trastornos funcionales, ahora denominados trastornos de interacción intestino-cerebro $(\mathrm{TIIC})^{5}$, que incluyen el estreñimiento entre sus síntomas predominantes, son el SII con estreñimiento, el estreñimiento funcional con sus tres subtipos (tránsito normal, tránsito lento y trastorno de evacuación) y el 


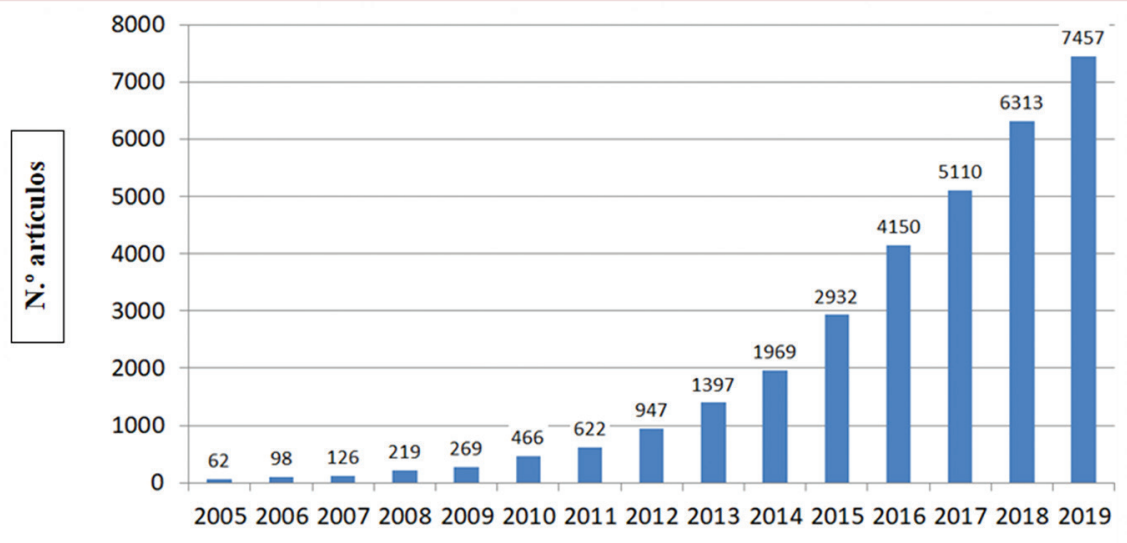

Años

Figura 1. Artículos sobre microbiota publicados en PubMed desde 2005 hasta 2019.

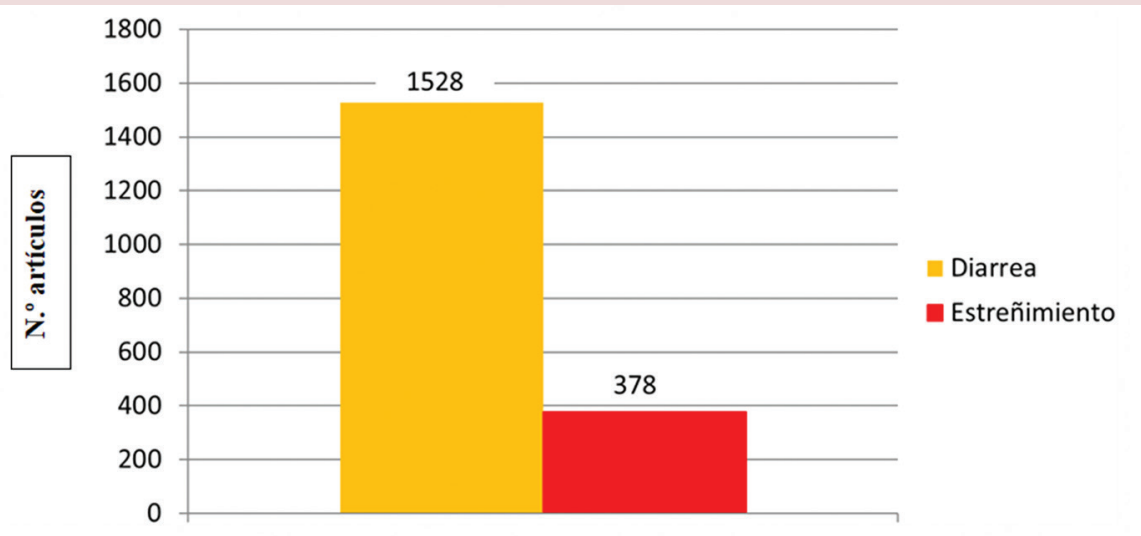

Figura 2. Artículos sobre microbiota en relación con el campo de estudio (diarrea o estreñimiento) hasta el año 2019.

estreñimiento inducido por opiáceos (categoría recientemente incluida $)^{5,6}$.

La microbiota parece influir directamente en la fisiopatogenia del SII y de los dos primeros subtipos de estreñimiento funcional, mientras que el estreñimiento inducido por opiáceos tiene en el consumo de estos fármacos como su factor detonante y en los pacientes con trastornos de la evacuación la etiología parece vincularse a alteraciones anatómicas y disfunción del piso de la pelvis que conducen a disinergia defecatoria ${ }^{5,6}$.

Se han publicado muchos artículos en los que se evidencia, mediante cultivos y la 
identificación de S16 rRNA, que existen diferencias en la composición de la microbiota intestinal de los pacientes estreñidos en comparación con los controles ${ }^{3}$. Parecería que los patrones de disbiosis de los pacientes estreñidos tienen menor diversidad y mayor abundancia de bacterias ${ }^{7}$. Si bien es cierto que varios estudios coinciden en que algunas especies de Clostridium y Veillonella se encuentran aumentadas ${ }^{8}$, u otras como Bifidobacterium y Lactobacillus se encuentran disminuidas, en los pacientes con estreñimiento ${ }^{9}$, no es posible establecer una especie metagenómica distintiva de este grupo de pacientes, ya que predominan distintos filotipos bacterianos en los diferentes estudios publicados ${ }^{3}$. Se han atribuido varias posibles causas a este fenómeno, entre las que destacan variaciones geográficas y étnicas, así como grupos etarios, resistencias antibióticas o consumo de fármacos en las distintas comunidades estudiadas $^{3,7}$. El estudio del metaboloma parece ser un parámetro más objetivo en este grupo de pacientes. Es así que en los estudios del aire espirado se evidencia una mayor concentración de metano en los pacientes estreñidos que en aquellos con diarrea o en los controles ${ }^{10,11}$; esto nos permite inferir que la presencia de arqueas metanogénicas, principalmente Methanobrevibacter smithii, es un componente determinante en la microbiota de los pacientes con este tipo de trastorno ${ }^{12,13}$. Otra forma indirecta de utilizar el metaboloma para analizar las variaciones de la microbiota de los pacientes con estreñimiento es la proporción de ácidos grasos de cadena corta (AGCC) en la materia fecal ${ }^{14}$. Un estudio controlado realizado en pacientes con SII mostró una concentración significativamente menor de AGCC, principalmente de butirato, en los pacientes con estreñimiento, en tanto que en los pacientes con diarrea la concentración fue significativamente mayor que en los controles ${ }^{15}$. Sin embargo, habría que analizar si estos hallazgos se relacionan con la producción de AGCC por parte de la microbiota intestinal o si son consecuencia de una mayor o menor absorción de estos por una disminución o un aumento de la motilidad, respectivamente $^{14}$.

También es importante mencionar el papel de la microbiota intestinal en el metabolismo de los ácidos biliares. Se sabe que las bacterias intestinales desconjugan los ácidos biliares primarios convirtiéndolos en secundarios, pero este proceso debe ser equilibrado ${ }^{16}$. La conjugación excesiva de ácidos biliares facilita su completa absorción en el yeyuno, con la consecuente disminución en la luz colónica; este fenómeno se relaciona con un aumento del tiempo de tránsito y estreñimiento ${ }^{17}$. Por el contrario, un exceso luminal de ácidos biliares induce una secreción aumentada de agua y electrolitos, y por tanto la aparición de diarrea $^{18}$.

\section{ESTRATEGIAS PARA LA MODULACIÓN DE LA MICROBIOTA INTESTINAL EN EL ESTREÑIMIENTO}

La manipulación de la microbiota es una opción dirigida a corregir la disbiosis intestinal con la intención de mejorar los síntomas de los TIIC $^{2,3}$. Las estrategias utilizadas con esta finalidad incluyen cambios en la dieta, prebióticos, probióticos, antibióticos y trasplante de microbiota fetal (TMF) ${ }^{3,4}$.

A continuación revisamos la evidencia disponible sobre la utilización de estas estrategias 
en el manejo de los trastornos funcionales con predominio de estreñimiento.

\section{Dieta}

Los cambios en la dieta son parte del tratamiento del estreñimiento funcional, pero es importante analizar cuáles de estas modificaciones tienen un efecto beneficioso sobre el tránsito intestinal y en qué casos este beneficio se relaciona con variaciones en la composición de la microbiota ${ }^{19}$. La suplementación con fibra es una de las opciones utilizadas en el manejo inicial de los pacientes con estreñimiento $^{20}$. Si bien es cierto que existen distintos productos que aportan fibra como suplemento dietético, parecería que el psyllium, fibra soluble derivada de Plantago ovata, ofrece los mayores beneficios. Un metaanálisis realizado en pacientes con SII mostró mejoría significativa de los síntomas en los estudios que utilizaron psyllium, y no en los que emplearon salvado $\mathrm{u}$ otros tipos de fibra como tratamiento $^{21}$. Adicionalmente, el psyllium parecería influir sobre la composición de la microbiota intestinal de los pacientes estreñidos. En un estudio contra placebo que incluyó 24 sujetos entre pacientes con estreñimiento y voluntarios sanos, se observó que, luego de un tratamiento de 7 días con psyllium, en el grupo con estreñimiento aumentó significativamente la proporción de Lachnospira, Roseburia y Faecalibacterium, géneros vinculados con la producción de butirato y el aumento de agua en la materia fecal ${ }^{22}$.

En los últimos años se ha incrementado el interés por el estudio de dietas específicas para el tratamiento de los trastornos digestivos funcionales, y la dieta baja en FODMAP (siglas en inglés de oligosacáridos, disacáridos, monosacáridos y polioles fermentables) es la más utilizada con esta finalidad ${ }^{23}$. Los FODMAP son pequeñas moléculas osmóticamente activas de lenta absorción intraluminal que son fermentadas con rapidez y dan lugar a la formación de hidrógeno, dióxido de carbono y metano ${ }^{24}$. En algunos estudios se ha reportado que una dieta baja en FODMAP puede modificar la composición de la microbiota intestinal, y se ha observado una disminución de las bifidobacterias en pacientes que siguieron esta dieta. En cuanto a la mejoría clínica de los pacientes que realizan una dieta baja en FODMAP, esta sería mayor en los que presentan diarrea que en aquellos con predominio de estreñimiento ${ }^{25}$. En 2018 se publicó un estudio en el que se comparó una dieta con prebióticos contra una dieta baja en FODMAP; ambas produjeron una mejoría de los síntomas, pero al suspenderlas el grupo de pacientes que recibió prebióticos mantuvo la mejoría y no así el grupo de dieta baja en FODMAP. En este estudio también se evidenció que el grupo de dieta baja en FODMAP experimentó una disminución de las bifidobacterias y un aumento de Bilophila wadsworthia, una proteobacteria productora de gas sulfhídrico relacionada con una mayor irritación del intestino ${ }^{26}$.

Basándonos en la evidencia presentada parecería que, entre los tipos de fibra disponibles, el psyllium otorga mayores beneficios desde el punto de vista clínico, así como también cambios favorables en la composición de la microbiota intestinal. En cuanto a la dieta baja en FODMAP, no parece haber evidencias categóricas que recomienden su utilización en los pacientes con trastornos funcionales con predominio de estreñimiento. 


\section{Probióticos}

Los probióticos son «microorganismos vivos» que, administrados en cantidades adecuadas, confieren un efecto beneficioso para la salud del huésped ${ }^{24}$. Se han usado ampliamente como cepas individuales o como parte de fórmulas multiespecie, y han demostrado que mejoran la función de barrera intestinal mediante un aumento de la secreción de mucina y una disminución de la muerte celular ${ }^{27}$. Estimulan el sistema inmunitario del huésped disminuyendo la inflamación y aumentando la respuesta inmunorreguladora. También son capaces de estimular la activación de mecanismos bactericidas, como la secreción de bacteriocinas y la liberación desde los enterocitos y las células de Paneth de péptidos antimicrobianos, como las defensinas y catelicidinas. Además, intervienen en la modulación de la motilidad intestinal, siendo así una alternativa potencialmente útil en el tratamiento de los pacientes con estreñimiento ${ }^{28}$.

A continuación presentamos la evidencia disponible más relevante con respecto a la utilización de probióticos, evaluando los cambios en la motilidad intestinal y los efectos en los TIIC. Evaluamos varios metaanálisis realizados tanto en pacientes adultos como en niños a partir de artículos que en su mayoría se detallan en la tabla 1.

La evidencia disponible para pacientes pediátricos es escasa y heterogénea. Entre las publicaciones destaca un metaanálisis centrado en países asiáticos realizado en el año 2017, que incluyó seis ensayos controlados aleatorizados. En este metaanálisis se evidenció una diferencia estadísticamente significativa en favor del uso de probióticos en cuanto a la frecuencia evacuatoria, pero la diferencia en la consistencia de la materia fecal no alcanzó significación estadística ${ }^{29}$. Estos resultados difieren de los obtenidos en una revisión sistemática realizada el mismo año con 515 pacientes incluidos en siete ensayos controlados aleatorizados, que mostró efectos beneficiosos en cuanto a la frecuencia evacuatoria solo en dos de los estudios, que utilizaron Lactobacillus reuteri DSM 17938 y Bifidobacterium longum 35624, respectivamente ${ }^{30}$. Tampoco un metaanálisis que incluyó 835 infantes de 17 ensayos controlados aleatorizados halló diferencias estadísticamente significativas para el uso de probióticos como terapia única o adyuvante en comparación con placebo $\mathrm{u}$ otros tratamientos en cuanto al aumento de la frecuencia evacuatoria y el éxito terapéutico en los pacientes estreñidos ${ }^{31}$.

La evidencia en población adulta tiene mayor peso. En el año 2013, Miller y Ouwehand ${ }^{32}$ publicaron un metaanálisis que incluyó 11 ensayos controlados aleatorizados con 464 sujetos, en el que se demostró que la administración de probióticos disminuye significativamente el tiempo de tránsito intestinal. Las conclusiones del estudio mostraron mayores beneficios en los sujetos ancianos y en aquellos que usaron cepas de Bifidobacterium lactis CNCM I-2494. En el año 2016, los mismos autores formaron parte de la realización de un nuevo metaanálisis ${ }^{33}$ que incluyó 15 ensayos controlados aleatorizados con 675 sujetos, en el que se ratificaron las conclusiones del estudio previamente publicado.

En el año 2014 se publicó un metaanálisis que evaluó los beneficios de la utilización de probióticos como tratamiento de pacientes con estreñimiento funcional. Se incluyeron 14 
Tabla 1. Ensayos controlados aleatorizados relevantes sobre probióticos en el tratamiento del síndrome del intestino irritable con estreñimiento y del estreñimiento funcional en adultos y niños

\begin{tabular}{|c|c|c|c|}
\hline Autor, año de publicación & Probiótico/preparación & Población & Resultado \\
\hline Koebnick, 2003 & L. casei Shirota & $\begin{array}{l}\text { Estreñimiento funcional } \\
\text { (adultos) }\end{array}$ & $\begin{array}{l}\text { Aumento de frecuencia evacuatoria } \\
\text { Disminución de consistencia de materia fecal }\end{array}$ \\
\hline Hongisto, 2006 & L. rhamnosus GG & $\begin{array}{l}\text { Estreñimiento funcional } \\
\text { (adultos) }\end{array}$ & No diferencia con placebo \\
\hline $\mathrm{Bu}, 2007$ & $\begin{array}{l}\text { L. casei } \\
\text { rhamnosus Lcr } 35\end{array}$ & $\begin{array}{l}\text { Estreñimiento funcional } \\
\text { (niños) }\end{array}$ & $\begin{array}{l}\text { Aumento de frecuencia evacuatoria } \\
\text { Disminución de consistencia de materia fecal }\end{array}$ \\
\hline Yang, 2008 & B. lactis DN-173 010 & $\begin{array}{l}\text { Estreñimiento funcional } \\
\text { (adultos) }\end{array}$ & $\begin{array}{l}\text { Aumento de frecuencia evacuatoria } \\
\text { Disminución de consistencia de materia fecal }\end{array}$ \\
\hline Agrawal, 2009 & B. lactis DN-173010 & SII-E (adultos) & Disminución de tiempo de tránsito colónico \\
\hline Holma, 2010 & L. rhamnosus GG & $\begin{array}{l}\text { Estreñimiento funcional } \\
\text { (adultos) }\end{array}$ & No diferencia con placebo \\
\hline Coccorullo, 2010 & L. reuteri DSM 17938 & $\begin{array}{l}\text { Estreñimiento funcional } \\
\text { (niños) }\end{array}$ & Aumento de frecuencia evacuatoria \\
\hline Krammer, 2011 & L. casei Shirota & Estreñimiento funcional (adultos) & No diferencia con placebo \\
\hline Guerra, 2011 & $\begin{array}{l}\text { B. longum } \\
\text { S. thermophilus }\end{array}$ & $\begin{array}{l}\text { Estreñimiento funcional } \\
\text { (niños) }\end{array}$ & Aumento de frecuencia evacuatoria \\
\hline Waller, 2011 & B. lactis HN019 & $\begin{array}{l}\text { Estreñimiento funcional } \\
\text { (adultos) }\end{array}$ & Disminución de tiempo de tránsito intestinal \\
\hline Waller, 2011 & B. lactis HN019 & $\begin{array}{l}\text { Estreñimiento funcional } \\
\text { (adultos) }\end{array}$ & Disminución de tiempo de tránsito intestinal \\
\hline Tabbers, 2011 & B. lactis DN-173010 & $\begin{array}{l}\text { Estreñimiento funcional } \\
\text { (niños) }\end{array}$ & No diferencia con placebo \\
\hline Ishizuka, 2012 & B. lactis GCL2505 & $\begin{array}{l}\text { Estreñimiento funcional } \\
\text { (adultos) }\end{array}$ & Aumento de frecuencia evacuatoria \\
\hline Riezzo, 2012 & L. paracasei & $\begin{array}{l}\text { Estreñimiento funcional } \\
\text { (adultos) }\end{array}$ & Disminución de consistencia de materia fecal \\
\hline Mazlyn, 2013 & L. casei Shirota & $\begin{array}{l}\text { Estreñimiento funcional } \\
\text { (adultos) }\end{array}$ & $\begin{array}{l}\text { Mejoría de sensación de evacuación } \\
\text { incompleta }\end{array}$ \\
\hline Favretto, 2013 & B. lactis & $\begin{array}{l}\text { Estreñimiento funcional } \\
\text { (adultos) }\end{array}$ & Aumento de frecuencia evacuatoria \\
\hline Bazzocchi, 2014 & $\begin{array}{l}\text { L. plantarum } \\
\text { L. acidophilus } \\
\text { L. rhamnosus } \\
\text { B. longum } \\
\text { B. breve }\end{array}$ & $\begin{array}{l}\text { Estreñimiento funcional } \\
\text { (adultos) }\end{array}$ & No diferencia con placebo \\
\hline Magro, 2014 & $\begin{array}{l}\text { L. acidophilus NCFM } \\
\text { B. lactis HN019 }\end{array}$ & $\begin{array}{l}\text { Estreñimiento funcional } \\
\text { (adultos) }\end{array}$ & No diferencia con placebo \\
\hline Mezzasalma, 2016 & $\begin{array}{l}\text { L. acidophilus } \\
\text { L. reuteri } \\
\text { L. plantarum } \\
\text { L. rhamnosus } \\
\text { B. lactis }\end{array}$ & SII-E (adultos) & $\begin{array}{l}\text { Mejoría de dolor abdominal } \\
\text { Aumento de frecuencia evacuatoria }\end{array}$ \\
\hline Wojtyniak, 2017 & L. casei rhamnosus Lcr35 & $\begin{array}{l}\text { Estreñimiento funcional } \\
\text { (niños) }\end{array}$ & No diferencia con placebo \\
\hline Yoon, 2018 & $\begin{array}{l}\text { S. thermophilus } \\
\text { L. plantarum }\end{array}$ & $\begin{array}{l}\text { SII-E/estreñimiento funcional } \\
\text { (adultos) }\end{array}$ & $\begin{array}{l}\text { Mejoría de calidad de vida } \\
\text { Disminución de consistencia de materia fecal }\end{array}$ \\
\hline
\end{tabular}

SII-E: síndrome del intestino irritable con estreñimiento.

ensayos controlados aleatorizados que reunieron 1182 individuos, en los que se observó que el uso de probióticos redujo significativamente el tiempo de tránsito intestinal total en 12.4 horas (intervalo de confianza del 95\% [IC 95\%]: -22.3 a -2.5$)$ y 
aumentó la frecuencia evacuatoria en 1.3 evacuaciones por semana (IC 95\%: 0.7 a 1.9). También se halló que el uso de probióticos mejoró significativamente la consistencia de la materia fecal. Sin embargo, estos hallazgos fueron significativos en los estudios que utilizaron B. lactis CNCM I-2494 como tratamiento, y no así en los que utilizaron Lactobacillus casei Shirota DN-114 00134.

El mismo año se publicó otro metaanálisis que también incluyó prebióticos y simbióticos (combinación de probióticos y prebióticos). Se analizaron 2575 sujetos incluidos en 43 ensayos controlados aleatorizados en pacientes con SII y estreñimiento funcional, y se observó que el tratamiento con probióticos y simbióticos aumentó significativamente el número de evacuaciones por semana en los pacientes estreñidos ${ }^{35}$.

Es importante destacar que no todos los probióticos parecen tener respuestas similares. En el año 2017 se publicó un metaanálisis que incluyó 21 ensayos controlados aleatorizados con 2656 sujetos con diagnóstico de estreñimiento funcional. Todos los estudios utilizaron especies de Lactobacillus o Bifidobacterium. El tratamiento con probióticos dio como resultado un aumento medio en la frecuencia de deposiciones semanales de 0.83 (IC 95\%: 0.53 a 1.14; $\mathrm{p}<0.001)$. Los efectos sobre la frecuencia evacuatoria también fueron significativos en los estudios en los que el estreñimiento funcional se diagnosticó utilizando los criterios de Roma II y Roma III ${ }^{36}$.

También en el año 2017 se publicó un metaanálisis de datos individuales de dos ensayos controlados aleatorizados que reunieron 579 sujetos con SII tratados con Saccharomyces cerevisiae CNCM I-3856. Este metaanálisis mostró una diferencia estadísticamente significativa en cuanto a la consistencia de la materia fecal en el subgrupo de pacientes con SII y predominio de estreñimiento ${ }^{37}$.

En el año 2018 se publicó un documento internacional de consenso sobre la utilidad de los probióticos en el tratamiento de los síntomas del tracto gastrointestinal bajo. Esta revisión, que incluyó 70 ensayos controlados aleatorizados (54 productos y 108 cepas), basándose en evidencias de nivel moderado concluyó que existen probióticos, especialmente bifidobacterias y lactobacilos, que aumentan la calidad de vida, reducen la distensión abdominal y mejoran la frecuencia y la consistencia de las evacuaciones en los pacientes estreñidos sin producir efectos adversos significativos, por lo que se consideran un tratamiento útil y seguro ${ }^{38}$.

De acuerdo con la evidencia disponible podemos mencionar que, pese a la heterogeneidad de los artículos publicados, algunos probióticos en cuya composición se incluyen especies de bifidobacterias y lactobacilos constituyen una alternativa útil y segura en el tratamiento del estreñimiento. Las evidencias más sólidas corresponden a los estudios que han utilizado cepas de B. lactis DN-173010, B. lactis HN019, L. reuteri y L. casei Shirota, tanto de manera individual como formando parte de combinaciones multiespecie.

\section{Prebióticos}

Los prebióticos son ingredientes alimentarios no digeribles que promueven la proliferación de bacterias gastrointestinales ${ }^{35}$. En su mayoría 
son FODMAP y se subdividen en disacáridos (como la lactulosa), oligosacáridos (como los fructooligosacáridos, los galactooligosacáridos, los isomaltooligosacáridos, los xilooligosacáridos, los trans-galactooligosacáridos y los oligosacáridos de soja) y polisacáridos (como la inulina, el almidón, la celulosa, la hemicelulosa y la pectina) ${ }^{24}$. De forma natural se encuentran en alimentos que en su mayoría contienen hidratos de carbono fermentables, como cereales, frutas, plátanos, espárragos, alcachofas, tomates, ajo, cebolla, linaza, avena, cebada y trigo. En algunos casos son producidos artificialmente, como la lactulosa, los galactooligosacáridos, los fructooligosacáridos, los maltooligosacáridos, las ciclodextrinas y la lactosacarosa ${ }^{24,35}$.

En cuanto a las evidencias disponibles, se han publicado algunos ensayos controlados aleatorizados que utilizaron inulina, trans-galactooligosacáridos y fructooligosacáridos, en los que se observan cambios evidentes en la composición de la microbiota, destacando el aumento de la proporción de bifidobacterias con la consecuente mayor generación de ácidos grasos de cadena corta ${ }^{24}$. De Vries, et al. ${ }^{39}$ realizaron una revisión sistemática y metaanálisis para evaluar los efectos de la suplementación con $\beta$-fructanos sobre la función intestinal a partir de 45 ensayos controlados aleatorizados que no mostraron heterogeneidad significativa entre sí. La variable primaria de desenlace fue la frecuencia de las evacuaciones, evaluada por el número de deposiciones al día durante el periodo de estudio. Los resultados mostraron que los fructooligosacáridos y la inulina ( $\beta$-fructanos de cadena corta) contribuyeron al aumento de la frecuencia de las evacuaciones de manera significativa (0.36 \pm 0.06 deposiciones al día; $\mathrm{p}<0.001)$. Estos hallazgos coinciden con los de un metaanálisis publicado en el año 2014 que incluyó datos de 252 sujetos en los que se evaluaron los efectos de la utilización de inulina en pacientes con diagnóstico de estreñimiento funcional crónico. Los autores encontraron efectos significativos de la inulina en la frecuencia de las deposiciones, la consistencia de las heces de acuerdo con la escala de Bristol y el tiempo de tránsito intestinal ${ }^{40}$.

La lactulosa es un disacárido utilizado en la práctica clínica desde 1957, entre cuyas principales aplicaciones se incluye el tratamiento del estreñimiento crónico ${ }^{41}$. Si bien en un principio se consideró que su mecanismo de acción estaba exclusivamente vinculado con su efecto osmótico, en la actualidad se conoce que este disacárido tiene también un efecto prebiótico, que se evidencia en los cambios de composición de la microbiota intestinal y el metaboloma posteriores a su administración ${ }^{24,41}$. En un estudio realizado con $26 \mathrm{mu}$ jeres sanas en Japón, Sakai, et al. ${ }^{42}$ observaron que tras la administración de 1, 2 o $3 \mathrm{~g}$ diarios de lactulosa durante 2 semanas aumentó de manera significativa el número de bifidobacterias en la materia fecal, así como también la frecuencia evacuatoria. Igualmente se observó este efecto en un ensayo controlado aleatorizado realizado en 65 pacientes con estreñimiento idiopático crónico, en el que Bouhnik, et al. ${ }^{43}$ evaluaron los efectos de la lactulosa y del polietilenglicol en la composición de la microbiota fecal. La eficacia clínica y la tolerabilidad fueron similares con ambos tratamientos, pero en el grupo de lactulosa se observó un aumento en el recuento de bifidobacterias fecales $(p=0.04)$.

El lactilol es un polialcohol (poliol) utilizado como endulzante artificial que al llegar al 
colon puede actuar como prebiótico al cambiar la composición de la microbiota aumentando el número de bifidobacterias ${ }^{44}$. Este poliol se ha utilizado en el tratamiento del estreñimiento funcional. En una revisión sistemática publicada en el año 2104 por Miller, et al. ${ }^{45}$, que incluyó 663 pacientes repartidos en 11 estudios, se encontró una mejoría significativa tanto en el número de evacuaciones por semana como en la consistencia de las heces.

\section{Antibióticos}

Es bien conocido que una microbiota intestinal productora de metano se relaciona con un mayor tiempo de tránsito intestinal, lo que puede vincularse con la presencia de estreñimiento funcional crónico y SII con predominio de estreñimiento ${ }^{10-13}$. Basándose en esta premisa se han realizado ensayos clínicos utilizando antibióticos con la intención de modificar la microbiota intestinal y, consecuentemente, disminuir el tiempo de tránsito ${ }^{3,14}$. Uno de los antibióticos utilizados con esta finalidad es la neomicina. Un estudio publicado en 2006 por Pimentel, et al. ${ }^{46}$ incluyó 39 pacientes con SII con estreñimiento. De estos, 20 recibieron placebo y 19 recibieron neomicina. El grupo tratado con neomicina mostró una mejoría global del $36.7 \pm 7.9 \%$, en comparación con el $5.0 \pm 3.2 \%$ del grupo placebo ( $\mathrm{p}<0.001$ ). Por otra parte, en 12 pacientes que mostraron una microbiota productora de metano en la prueba de aliento y todos ellos reportaron estreñimiento, la mejoría del estreñimiento fue significativamente mayor en los que recibieron neomicina $(44.0 \pm 12.3 \%)$ que en los que tomaron placebo $(5.0 \pm 5.1 \%)$ $(\mathrm{p}<0.05)^{46}$.
Otro antibiótico estudiado para el manejo del estreñimiento es la lincomicina. En un estudio contra placebo publicado por Bustos Fernández, et al. $^{47}$, realizado en 30 pacientes estreñidos tratados con dieta rica en fibra, se observó que agregar lincomicina en una dosis diaria de 500 mg aumentó de manera significativa la frecuencia evacuatoria y la consistencia de las heces.

La rifaximina es un antibiótico oral no absorbible de amplio espectro. Se han publicado estudios que sugieren que disminuye la respuesta inflamatoria del huésped y restablece la diversidad de la microbiota. También es conocido su efecto contra el sobrecrecimiento bacteriano del intestino delgado ${ }^{48}$. La acción de la rifaximina en los pacientes con microbiota productora de metano parece ser menos efectiva $^{14}$.

En el año 2010, Low, et al. ${ }^{49}$ publicaron un estudio retrospectivo en el que revisaron la historia clínica de los pacientes con microbiota metanogénica en una prueba de aire espirado con lactulosa. Los pacientes recibieron tratamiento con neomicina (500 mg b.i.d. durante 10 días), rifaximina (400 $\mathrm{mg}$ t.i.d. durante 10 días) o una combinación de ambos (durante 10 días). De los sujetos que recibieron el tratamiento combinado, el $85 \%$ tuvo mejoría clínica en comparación con el 63\% de los sujetos en el grupo de neomicina $(p=0.15)$ y el 56\% de los sujetos en el grupo de rifaximina $(p=0.01)$. La diferencia entre los grupos de neomicina y rifaximina no fue significativa. Al evaluar los resultados de la erradicación del metano, el $87 \%$ de los sujetos que tomaron la combinación de rifaximina y neomicina erradicaron el metano en su prueba de aliento, frente al 33\% de los sujetos en el 
grupo de neomicina ( $p=0.001)$ y apenas el $28 \%$ en el grupo de rifaximina $(p=0.001)$.

En el año 2014, Pimentel, et al. ${ }^{50}$ publicaron un ensayo controlado aleatorizado con 31 pacientes de entre 18 y 65 años con diagnóstico de SII con estreñimiento y prueba del aire espirado con hiperproducción de metano. Los sujetos fueron aleatorizados para recibir neomicina y placebo $(n=16)$ o neomicina y rifaximina $(n=15)$ durante 14 días. La gravedad del estreñimiento fue significativamente menor en el grupo de neomicina y rifaximina $(28.6 \pm 30.8)$ que en el de neomicina sola (61.2 $\pm 24.1)(p=0.0042)$, con una respuesta significativamente mayor en la resolución del estreñimiento ( $p=0.007$ ), el esfuerzo evacuatorio $(\mathrm{p}=0.017)$ y la hinchazón ( $\mathrm{p}=0.020)$, pero no en el dolor abdominal. En el grupo de neomicina y rifaximina, los sujetos que redujeron los valores de metano después del tratamiento informaron una gravedad del estreñimiento significativamente menor (30.5 \pm 21.8) que los sujetos con metano persistentemente elevado $(67.2 \pm 32.1)(p=0.020)$. Por $s u$ parte, Goshal, et al. ${ }^{51}$ publicaron en el año 2018 un ensayo controlado aleatorizado que realizaron con el objetivo de evaluar la respuesta al tratamiento con rifaximina en cuanto a la normalización del tiempo de tránsito y la mejoría de los síntomas de estreñimiento según la reducción de la eliminación de metano. Se evaluaron la forma de las heces (Escala de Bristol), la frecuencia evacuatoria, el tiempo de tránsito colónico y la producción de metano en el aliento en 23 pacientes con estreñimiento (10 con SII y 13 con estreñimiento funcional). Los pacientes estreñidos hiperproductores de metano (basal $\geq 10$ p.p.m. o aumento tras la administración de lactulosa > 10 p.p.m.) fueron aleatorizados para recibir rifaximina (400 $\mathrm{mg}$ t.i.d. durante 2 semanas) o placebo. La rifaximina redujo significativamente la producción de metano en comparación con el grupo que recibió placebo. Así mismo, el tiempo de tránsito colónico, la forma de las heces y la frecuencia evacuatoria mejoraron de manera significativa en el grupo de rifaximina.

De acuerdo con la evidencia revisada, parece claro que el uso de determinados antibióticos influye significativamente en la composición de la microbiota intestinal y en la producción de metano, así como en la mejoría de los síntomas, de los pacientes estreñidos. Si bien parecería que la combinación de rifaximina y neomicina es la alternativa más eficaz, los ensayos realizados con rifaximina sola también muestran resultados alentadores en este tipo de pacientes; sin embargo, el número de publicaciones es aún escaso como para recomendar un esquema específico de manera categórica.

\section{Trasplante de microbiota fecal}

Se han publicado varios artículos con resultados favorables utilizando TMF en pacientes con estreñimiento. En ellos se observa un aumento de la frecuencia evacuatoria, una mejoría de la consistencia de la materia fecal y una disminución del tiempo de tránsito colónico y de la sensación de evacuación incompleta; no obstante, son estudios con pocos pacientes y en su mayoría son no controla$\operatorname{dos}^{3}$. En un estudio controlado realizado por Tian, et al. ${ }^{52}$ durante 12 semanas en pacientes con estreñimiento funcional se comparó la evolución de 30 pacientes que recibieron TMF con la de 30 pacientes que recibieron laxantes 
convencionales. El grupo de TMF mejoró significativamente la frecuencia y la consistencia de las deposiciones, así como el tiempo de tránsito colónico y la tasa de mejoría clínica. Por su parte, Ding, et al. ${ }^{53}$ publicaron una serie de 52 casos de pacientes estreñidos que recibieron TMF y fueron seguidos durante 24 semanas, obteniendo mejoría significativa en cuanto a frecuencia evacuatoria, consistencia de la materia fecal, disminución del tiempo de tránsito y mejoría de la sensación de evacuación incompleta.

Los resultados de los estudios publicados ubican al TMF como una alternativa promisoria en el tratamiento del estreñimiento funcional, pero es necesario contar con estudios controlados más grandes para poder recomendarlo de forma absoluta.

En relación con el SII se han publicado tres metaanálisis que han evaluado el TMF como tratamiento. El primero de ellos, realizado por Myneedu, et al. ${ }^{54}$, incluyó ocho estudios no controlados que sumaban 90 sujetos y cinco ensayos controlados aleatorizados con 151 pacientes y 105 controles. Si bien los en los estudios no controlados se observó mejoría clínica, en los ensayos controlados aleatorizados las diferencias no fueron significativas, por lo que los autores concluyen que el TMF no fue efectivo como tratamiento del SII. Estos resultados son parecidos a los obtenidos por $\mathrm{Xu}$, et al. ${ }^{55}$, que incluyeron en su metaanálisis 254 participantes de cuatro ensayos controlados aleatorizados. Estos autores concluyen que el TMF no fue beneficioso en cuanto a la mejoría global de los síntomas del SII. El último de los metaanálisis publicados es el de Ianiro, et al. ${ }^{56}$, quienes analizaron los datos de 267 pacientes participantes en cinco ensayos controlados aleatorizados; cabe señalar que apenas el $7.8 \%$ de los pacientes eran del subtipo con estreñimiento. El análisis global de los resultados no mostró diferencias significativas contra placebo en favor del tratamiento.

Es importante recalcar que los tres metaanálisis reportaron dificultades para la elaboración debido a la heterogeneidad de los artículos incluidos. Las diferentes vías de administración (cápsulas, sonda nasoyeyunal o colonoscopía), así como la falta de una diferenciación clara de varios de los artículos incluidos en relación con el subtipo de SII de los pacientes estudiados, impiden establecer conclusiones categóricas, y son necesarios más ensayos con protocolos específicos para futuros análisis ${ }^{54-56}$.

\section{CONCLUSIÓN}

La evidencia sobre la importancia de la composición de la microbiota intestinal en los TIIC ha tenido un crecimiento exponencial en los últimos años, y parecería lógico relacionar la presencia de cambios en la flora intestinal con la aparición de trastornos como el SII con estreñimiento y el estreñimiento funcional.

Sin embargo, existen todavía muchas preguntas fisiopatológicas por contestar y son necesarias futuras investigaciones para definir aspectos tales como si el aumento de la flora metanogénica es causa o consecuencia, si los pacientes tienen aumento de este tipo de microorganismos por tener un tránsito lento o si el tránsito lento es consecuencia del aumento de este tipo de flora. 
En relación con las intervenciones dirigidas a la modulación de la microbiota intestinal, se sabe que la implementación de cambios en la dieta, el uso de prebióticos, probióticos o antibióticos, y el TMF producen variaciones en este complejo ecosistema. Si bien estas intervenciones se realizan con la intención de que el aumento o la disminución de diferentes cepas microbianas produzcan cambios en la sintomatología de los pacientes con estreñimiento, no todas estas estrategias han mostrado resultados satisfactorios desde el punto de vista clínico.

El uso de psyllium produce cambios en la composición de la microbiota intestinal y parece mejorar los síntomas de los pacientes con SII con estreñimiento.

La utilización de rifaximina ha mostrado eficacia en el tratamiento de los síntomas de los pacientes con SII con estreñimiento. También, cuando se utiliza en combinación con neomicina, parece disminuir el tiempo de tránsito intestinal de los pacientes con estreñimiento funcional.

El uso de prebióticos, como inulina, lactulosa y lactilol, ha mostrado efectos beneficiosos en la frecuencia de las deposiciones, la consistencia de las heces y el tiempo de tránsito intestinal en los pacientes con estreñimiento funcional. En el caso específico de la lactulosa existe además evidencia suficiente que respalda que su utilización promueve cambios favorables en la composición de la microbiota intestinal de este grupo de pacientes.

El tratamiento con probióticos, principalmente con las especies B. lactis DN-173010, B. lactis HN019, L. reuteri y L. casei Shirota, o combinaciones de ellas, parece ser efectivo como estrategia para mejorar los síntomas de los pacientes con SII con estreñimiento y también de los que presentan estreñimiento funcional.

A pesar de que distintas intervenciones sobre la modulación de la microbiota intestinal parecen ser efectivas en el manejo del SII con estreñimiento y del estreñimiento funcional, se necesitan estudios con mayor número de pacientes y menos heterogéneos para establecer recomendaciones de manera absoluta.

\section{CONFLICTO DE INTERESES}

Los autores declaran no tener conflictos de intereses en el momento de la elaboración de este artículo.

\section{FINANCIACIÓN}

Ninguna.

\section{RESPONSABILIDADES ÉTICAS}

Protección de personas y animales. Los autores declaran que para esta investigación no se han realizado experimentos en seres humanos ni en animales.

Confidencialidad de los datos. Los autores declaran que en este artículo no aparecen datos de pacientes.

Derecho a la privacidad y consentimiento informado. Los autores declaran que en este artículo no aparecen datos de pacientes. 


\section{BIBLIOGRAFÍA}

1. Bäckhed F, Fraser C, Ringel Y, Sanders ME, Sartor RB, Sherman PM, et al. Defining a healthy human gut microbiome: current concepts, future directions, and clinical applications. Cell Host Microbe. 2012;12:611-22.

2. Rodiño-Janeiro B, Vicario M, Alonso-Cotoner C, Pascua-García R, Santos J. A review of microbiota and irritable bowel syndrome: future in therapies. Adv Ther. 2018;35:289-310.

3. Ohkusa T, Koido S, Nishikawa Y, Sato N. Gut microbiota and chronic constipation: a review and update. Front Med (Lausanne). 2019;6:19.

4. Villar-Chávez A, Coss-Adame E. Nuevos tratamientos para el estreñimiento crónico: ¿cuáles son las evidencias? NeuroGastroLatam Rev. 2017;1:42-6.

5. Lacy B, Mearin F, Chang L, Chey W, Lembo A, Simren M, et al. Rome IV diagnostic criteria for functional gastrointestinal disorders. Bowel Disorders Gastroenterology. 2016;150:1393-407.

6. Solé L. Trastornos funcionales de la evacuación. Una revisión. NeuroGastroLatam Rev. 2017;1:187-205.

7. Quigley EM, Spiller RC. Constipation and the microbiome: lumen versus mucosa! Gastroenterology. 2016;150:300-3.

8. Malinen E, Rinttilä T, Kajander K, Mättö J, Kassinen A, Krogius L, et al. Analysis of the fecal microbiota of irritable bowel syndrome patients and healthy controls with real-time PCR. Am J Gastroenterol. 2005;100:373-82.

9. Zoppi G, Cinquetti M, Luciano A, Benini A, Muner A, Bertazzoni Minelli E. The intestinal ecosystem in chronic functional constipation. Acta Paediatr. 1998;87:836-41.

10. Pichetshote N, Rezaie A. Breath tests for functional gastrointestinal disorders: when and for what? NeuroGastroLatam Rev. 2018;2:87-97.

11. Chatterjee S, Park S, Low K, Kong Y, Pimentel M. The degree of breath methane production in IBS correlates with the severity of constipation. Am J Gastroenterol. 2007;102:837-41.

12. Kim G, Deepinder F, Morales W, Hwang L, Weitsman S, Chang C, et al. Methanobrevibacter smithii is the predominant methanogen in patients with constipation-predominant IBS and methane on breath. Dig Dis Sci. 2012;57:3213-8.

13. Ghoshal U, Shukla R, Srivastava D, Ghoshal UC. Irritable bowel syndrome, particularly the constipation-predominant form, involves an increase in Methanobrevibacter smithii, which is associated with higher methane production. Gut Liver. 2016;10:932-8.

14. Dass N, John A, Bassil A, Crumbley C, Shehee W, Maurio F, et al. The relationship between the effects of short-chain fatty acids on intestinal motility in vitro and GPR43 receptor activation. Neurogastroenterol Motil. 2007;19:66-74.

15. Sun Q, Jia Q, Song L, Duan L. Alterations in fecal short-chain fatty acids in patients with irritable bowel syndrome: a systematic review and metaanalysis. Medicine (Balt). 2019;98:e14513.

16. Ramírez-Pérez O, Cruz-Ramón V, Chinchilla-López P, Méndez-Sánchez N. The role of the gut microbiota in bile acid metabolism. Ann Hepatol. 2017;16(Suppl 1):s15-20.

17. Vijayvargiya P, Busciglio I, Burton D, Donato L, Lueke A, Camilleri M. Bile acid deficiency in a subgroup of patients with irritable bowel syndrome with constipation based on biomarkers in serum and fecal samples. Clin Gastroenterol Hepatol. 2018;16:522-7.

18. Peleman C, Camilleri M, Busciglio I, Burton D, Donato L, Zinsmeister AR. Colonic transit and bile acid synthesis or excretion in patients with irritable bowel syndrome-diarrhea without bile acid malabsorption. Gastroenterol Hepatol. 2017;15:720-7.e1.

19. Okawa Y, Fukudo S, Sanada H. Specific foods can reduce symptoms of irritable bowel syndrome and functional constipation: a review. Biopsychosoc Med. 2019;13:10.

20. Axelrod C. Comparison of the role of fiber in the treatment of functional constipation in children and adults. NeuroGastroLatam Rev. 2018;2:149-56.

21. Ford AC, Talley NJ, Spiegel BM, Foxx-Orenstein AE, Schiller L, Quigle$y \mathrm{EM}$, et al. Effect of fibre, antispasmodics, and peppermint oil in the treatment of irritable bowel syndrome: systematic review and meta-analysis. BMJ. 2008;337:a2313.
22. Jalanka J, Major G, Murray K, Singh G, Nowak A, Kurtz C, et al. The effect of psyllium husk on intestinal microbiota in constipated patients and healthy controls. Int J Mol Sci. 2019;20:433.

23. Pourmand H, Esmaillzadeh A. Consumption of a low fermentable oligo-, di-, mono-saccharides, and polyols diet and irritable bowel syndrome: a systematic review. Int J Prev Med. 2017;8:104.

24. Bustos-Fernández LM, Hanna-Jairala I. Tratamiento actual del síndrome de intestino irritable. Una nueva visión basada en la experiencia y la evidencia. Acta Gastroenterol Latinoam. 2019;49:381-93.

25. Staudacher HM, Lomer MC, Anderson, JL. Fermentable carbohydrate restriction reduces luminal bifidobacteria and gastrointestinal symptoms in patients with irritable bowel syndrome. J Nutrt. 2012;142:1510-8.

26. Huaman JW, Mego M, Manichanh C, Cañellas N, Cañueto D, Segurola H, et al. Effects of prebiotics vs. diet low in FODMAPs in patients with functional gut disorders. Gastroenterol. 2018;155:1004-7.

27. Bull M, Plummer N. Part two: Treatments for chronic gastrointestinal diseases and gut dysbiosis. Integr Med (Encinitas). 2015;14:25-33.

28. Quigley E. Probiotics in irritable bowel syndrome. NeuroGastroLatam Rev. 2017;1:4-11.

29. Huang R, Hu J. Positive effect of probiotics on constipation in children: a systematic review and meta-analysis of six randomized controlled trials. Front Cell Infect Microbiol. 2017;7:153.

30. Wojtyniak K, Szajewska H Systematic review: probiotics for functional constipation in children. Eur J Pediatr. 2017;176:1155-62.

31. Harris RG, Neale EP, Ferreira I. When poorly conducted systematic reviews and meta-analyses can mislead: a critical appraisal and update of systematic reviews and meta-analyses examining the effects of probiotics in the treatment of functional constipation in children. Am J Clin Nutr. 2019;110:177-95.

32. Miller LE, Ouwehand AC Probiotic supplementation decreases intestinal transit time: meta-analysis of randomized controlled trials. World J Gastroenterol. 2013 Aug 7;19(29):4718-25.

33. Miller LE, Ouwehand AC Contemporary meta-analysis of short-term probiotic consumption on gastrointestinal transit. World J Gastroenterol. 2016;22:5122-31.

34. Dimidi E, Christodoulides S, Fragkos KC, Scott SM, Whelan K. The effect of probiotics on functional constipation in adults: a systematic review and metaanalysis of randomized controlled trials. Am J Clin Nutr. 2014;100:1075-84.

35. Ford AC, Quigley EM, Lacy BE, Lembo AJ, Saito YA, Schiller LR, et al. Efficacy of prebiotics, probiotics, and synbiotics in irritable bowel syndrome and chronic idiopathic constipation: systematic review and meta-analysis. Am J Gastroenterol. 2014;109:1547-61.

36. Miller LE, Ouwehand AC, Ibarra A. Effects of probiotic-containing products on stool frequency and intestinal transit in constipated adults: systematic review and meta-analysis of randomized controlled trials. Ann Gastroenterol. 2017;30:629-39.

37. Cayzeele-Decherf A, Pélerin F, Leuillet S, Douillard B, Housez B, Cazaubiel M, et al. Saccharomyces cerevisiae CNCM I-3856 in irritable bowel syndrome: an individual subject meta-analysis. World J Gastroenterol. 2017;23:336-44.

38. Hungin A, Mitchell CR, Whorwell P, Mulligan C, ColeO, Agréus L, et al.;European Society for Primary Care Gastroenterology. Systematic review: probiotics in the management of lower gastrointestinal symptoms - an updated evidence-based international consensus. Aliment Pharmacol Ther. 2018;47:1054-70

39. de Vries J, Le Bourgot C, Calame W, Respondek F. Effects of $\beta$-fructans fiber on bowel function: a systematic review and meta-analysis. Nutrients. 2019;11:91.

40. Collado Yurrita L, San Mauro Martín I, Ciudad-Cabañas MJ, CallePurón ME, Hernández Cabria M. Effectiveness of inulin intake on indicators of chronic constipation; a meta-analysis of controlled randomized clinical trials. Nutr Hosp. 2014;30:244-52.

41. Ruszkowski J, Witkowski JM. Lactulose: patient- and dose-dependent prebiotic properties in humans. Anaerobe. 2019;59:100-6.

42. Sakai Y, Seki N, Hamano H, Ochi H, Abe F, Shimizu F, et al. A study of the prebiotic effect of lactulose at low dosages in healthy Japanese women. Biosci Microbiota Food Health. 2019;38:69-72. 
43. Bouhnik Y, Neut C, Raskine L, Michel C, Riottot M, Andrieux C, et al. Prospective, randomized, parallelgroup trial to evaluate the effects of lactulose and polyethyleneglycol-4000 on colonic flora in chronic idiopathic constipation. Aliment Pharmacol Ther. 2004;19:889-99.

44. Ruiz-Ojeda FJ, Plaza-Díaz J, Sáez-Lara MJ, Gil A. Effects of sweeteners on the gut microbiota: a review of experimental studies and clinical trials. Adv Nutr. 2019;10(Suppl 1):S31-S48.

45. Miller LE, Tennilä J, Ouwehand AC. Efficacy and tolerance of lactitol supplementation for adult constipation: a systematic review and metaanalysis. Clin Exp Gastroenterol. 2014;7:241-8.

46. Pimentel M, Chatterjee S, Chow EJ, Park S, Kong Y. Neomycin improves constipation-predominant irritable bowel syndrome in a fashion that is dependent on the presence of methane gas: subanalysis of a doubleblind randomized controlled study. Dig Dis Sci. 2006;51:1297-301.

47. Bustos Fernández LM, Prizont R, Soifer LO. A controlled pilot study on the efficacy of a low dose antibiotic for the treatment of chronic constipation in patients receiving a high fiber diet. Acta Gastroenterol Latinoam. 2013;43:275-8.

48. Pimentel M, Barlow GM. Rifaximin in the treatment of irritable bowel syndrome. NeuroGastroLatam Rev. 2017;1:91-100.

49. Low K, Hwang L, Hua J, Zhu A, Morales W, Pimentel M. A combination of rifaximin and neomycin is most effective in treating irritable bowel syndrome patients with methane on lactulose breath test. J Clin Gastroenterol. 2010;44:547-50.
50. Pimentel M, Chang C, Chua KS, Mirocha J, DiBaise J, Rao S, et al. Antibiotic treatment of constipation-predominant irritable bowel syndrome. Dig Dis Sci. 2014;59:1278-85.

51. Ghoshal UC, Srivastava D, Misra A. A randomized double-blind placebocontrolled trial showing rifaximin to improve constipation by reducing methane production and accelerating colon transit: a pilot study. Indian J Gastroenterol. 2018;37:416-23.

52. Tian H, Ge X, Nie Y, Yang L, Ding C, McFarland LV, et al. Fecal microbiota transplantation in patients with slow-transit constipation: a randomized, clinical trial. PLoS One. 2017;12:e0171308.

53. Ding C, Fan W, Gu L, Tian H, Ge X, Gong J, et al. Outcomes and prognostic factors of fecal microbiota transplantation in patients with slow transit constipation: results from a prospective study with longterm follow-up. Gastroenterol Rep. 2018;6:101-7.

54. Myneedu K, Deoker A, Schmulson M, Bashashati M. Fecal microbiota transplantation in irritable bowel syndrome: a systematic review and metaanalysis. United European Gastroenterol J. 2019;7:1033-41.

55. Xu D, Chen VL, Steiner CA, Berinstein JA, Eswaran S, Waljee AK, et al. Efficacy of fecal microbiota transplantation in irritable bowel syndrome: a systematic review and meta-analysis. Am J Gastroenterol. 2019;114:1043-50.

56. Ianiro G, Eusebi LH, Black CJ, Gasbarrini A, Cammarota G, Ford AC. Systematic review with meta-analysis: efficacy of faecal microbiota transplantation for the treatment of irritable bowel syndrome. Aliment Pharmacol Ther. 2019;50:240-8. 